\title{
Great Efficacy of Afatinib in a Patient with Lung Adenocarcinoma Harboring EGFR L833V/H835L Mutations: A Case Report
}

This article was published in the following Dove Press journal: OncoTargets and Therapy

Xiang Long'

Tian Qin ${ }^{2}$

Junhong $\operatorname{Lin}^{3}$

'Department of Respiratory Medicine, Peking University Shenzhen Hospital, Shenzhen, 518036, China; ${ }^{2}$ Burning Rock Biotech, Guangzhou, 510300, China; ${ }^{3}$ Department of Respiratory Medicine, Huizhou Municipal Central Hospital, Huizhou, 51600I, China
Correspondence: Junhong Lin

Department of Respiratory Medicine, Huizhou Municipal Central Hospital, No. 4I, West Eling Road, Huicheng District, Huizhou 51600I, China

Email hz_ljh@I63.com

\begin{abstract}
Uncommon mutations account for 10-15\% of epidermal growth factor receptor (EGFR) mutations in patients with non-small-cell lung cancer (NSCLC). Most of them are proved to be sensitive or resistant to EGFR-tyrosine kinase inhibitors (TKIs). However, there is insufficient evidence for other less common types of EGFR mutations, such as complex mutations. Here, we present a 65-year-old never-smoking male who was diagnosed with stage IV lung adenocarcinoma. A rare L833V/H835L complex mutation in exon 21 of EGFR was detected in plasma and pleural effusion by next generation sequencing (NGS). Afatinib was used as first-line therapy and showed very good efficacy. To date, the patient is still benefited from afatinib treatment for a total of 10 months, with no signs of disease progression. Our case suggests that a comprehensive screening for EGFR mutations should be conducted before treatment in clinical practice, and afatinib could be a first-line treatment option in NSCLC patients harboring H833V/H835L mutations.
\end{abstract}

Keywords: non-small-cell lung cancer, epidermal growth factor receptor, afatinib, EGFR compound mutation, L833V/H835L

\section{Introduction}

Epidermal growth factor receptor (EGFR) is the most common driver gene in nonsmall cell lung cancer (NSCLC) patients. The mutations in exons 18 to 21, especially exon 19 deletion and exon 21 L858R account for a large proportion of all EGFR mutations. ${ }^{1}$ In recent years, with the application of the next generation sequencing (NGS), more and more uncommon EGFR mutations have been detected, which may potentially benefit from EGFR TKIs. ${ }^{2-4}$ However, more evidence should be provided for the clinical significance of rare EGFR mutations, especially complex mutations. Here, we present a patient with NSCLC harboring EGFR exon $21 \mathrm{~L} 833 \mathrm{~V} / \mathrm{H} 835 \mathrm{~L}$ complex mutation, who had clinical response to first-line afatinib therapy.

\section{Case Report}

A 65-year-old never-smoking male patient presented to our hospital in September, 2019 due to 1-month history of cough and a few days of thoracalgia. According to the subsequently high resolution computed tomography (HRCT) scanning and pathological examination, the patient was diagnosed with stage IV lung adenocarcinoma. For genomic analysis, capture-based ultra-deep targeted sequencing (depth: 
10,000×, LOD: $0.2 \%$ ) was performed on circulating tumor DNA (ctDNA) extracted from pleural effusion and plasma by a large panel consisting of all exons and critical introns of 520 cancer-related genes (Oncosreen Plus ${ }^{\mathrm{TM}}$, Burning Rock Biotech. Guangzhou, China). The results showed that the patient only had a complex EGFR mutation: L833V/H835L in exon 21 (VAF: 6.72\%. Figure 1). The tumor mutation burden (TMB) was 2.4 mutations/Mb and the expression of PD-L1 was negative. After literature research and multiple disciplinary team (MDT) discussion, daily oral of afatinib (40mg, bid.) was administered starting at the middle of September 2019. No grade III or IV adverse event (AE) was observed. Surprisingly, after one month of treatment, HRCT scan revealed a significant shrinkage in the nodules on the middle right lobe of the lung and decrease in number of nodules throughout the lung as compared to the baseline, indicating a partial response to afatinib (Figure $2 \mathrm{~A}$ and $\mathrm{B}$ ). In addition, no mutations were detected in plasma DNA using the previous NGS panel, also suggesting a good efficacy of afatinib. In December 2019, no apparent abnormalities were found by color ultrasound of neck and abdomen, and the level of circulating carcinoembryonic antigen (CEA) was significantly decreased compared with the

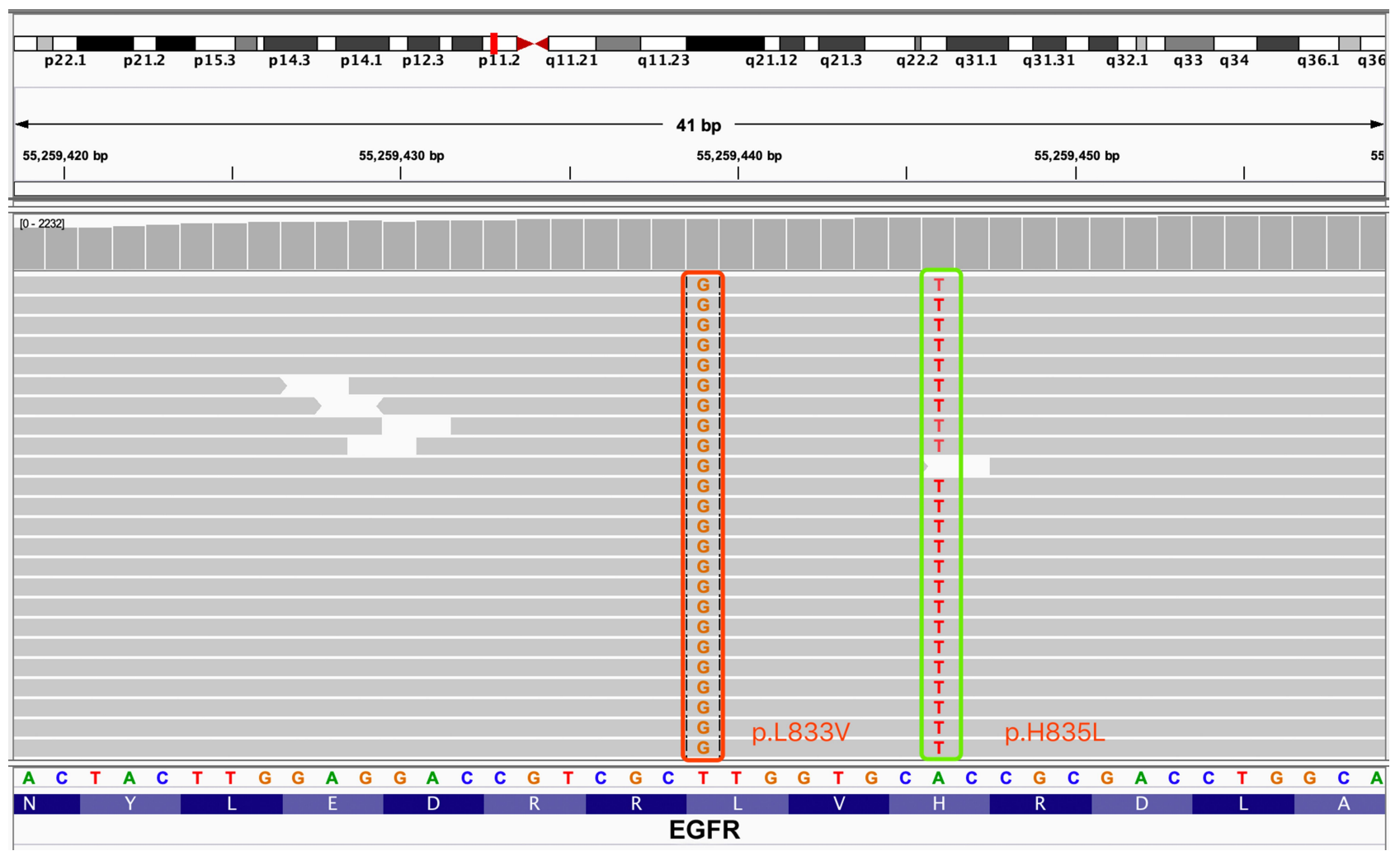

Figure I Identification of L833V and H835L in EGFR exon 2 I from liquid biopsy.
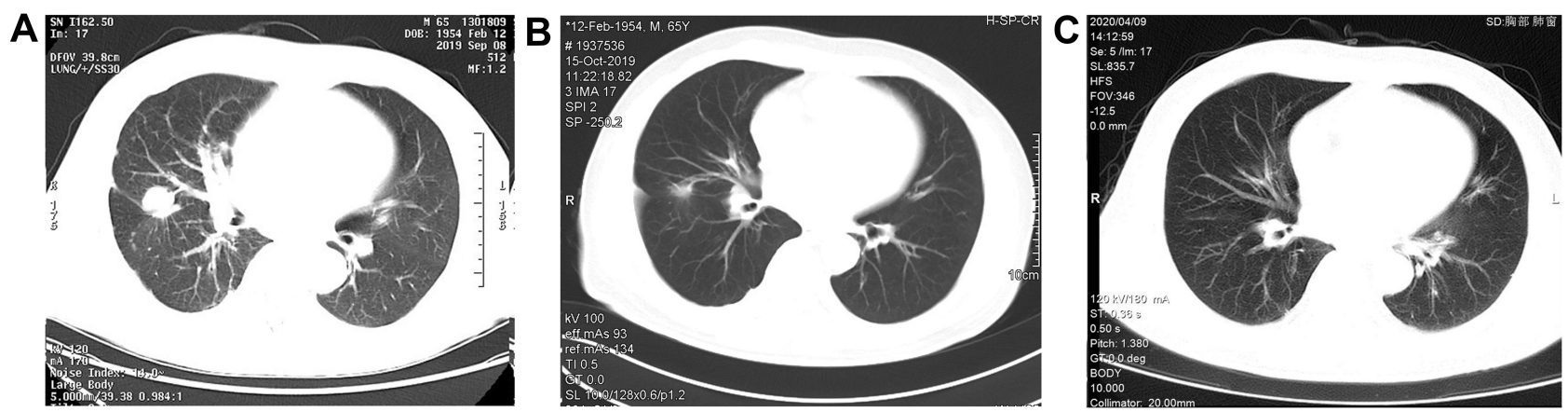

Figure 2 Medical imaging. CT scan with a right middle lobe lung mass before $(\mathbf{A})$ and after (B) I month and 7 month $(\mathbf{C})$ of afatinib treatment. 
first two times. After 7 months of afatinib treatment, the nodule in the middle lobe of right lung was almost disappeared (Figure 2A and $\mathrm{C}$ ). No metastasis was found and the mutation in ctDNA remained negative. The patient is still benefit from afatinib treatment at the last follow-up time (July 20, 2020). The PFS of afatinib was over 10 months.

\section{Discussion}

In the present case, we showed the clinical efficacy of afatinib in a patient with EGFR L833V/H835L mutations. The patient is still under treatment with afatinib, and the PFS is over 10 months. To the best of our knowledge, this is the first case of first-line treatment with afatinib in Asian NSCLC patient harboring EGFR L388V/H835L mutation.

Complex EGFR mutation is a small group of uncommon mutations, which comprised of two or more EGFR point mutations. Among them, the most frequent type is an EGFR sensitive mutation in combination with a nonclassical mutation, which is called compound mutation. ${ }^{5-}$

7 Another frequent type is EGFR sensitive mutation plus resistant mutation, such as L858R/T790M, 19del/T790M and T790M/C797S. ${ }^{8}$ Two or more synchronous rare EGFR mutations like L833V/H835L mutations are extremely rare in lung cancer. A previous study which compared the testing methods and tissue types of EGFR detection have found $3 \mathrm{~L} 833 \mathrm{~V} / \mathrm{H} 835 \mathrm{~L}$ mutations in $157 \mathrm{NSCLC}$ patients. ${ }^{9}$ On the other hand, only a few studies have detailed the clinical significance of this mutation. An Asian patient harboring $\mathrm{L} 833 \mathrm{~V} / \mathrm{H} 835 \mathrm{~L}$ mutations were reported to benefit from gefitinib treatment lasting 34 weeks. ${ }^{10}$ In addition, a recent cohort study about in cis EGFR mutations in NSCLC has reported a case carrying L833V/H835L mutations. The patient was treated with gefitinib and the PFS was over 15 months. ${ }^{11}$ Qin et al have reported a Chinese patient with advanced lung adenocarcinoma harboring a rare triple EGFR mutation (L833V/H835L/R670W) was resistant to gefitinib but sensitive to afatinib, suggesting that R670W may contribute to resistance to first-generation EGFR TKIs. ${ }^{12}$ Frega et al presented an Italian patient with advanced NSCLC harboring synchronous EGFR L833V/H835L/E709K mutations who achieved partial response after 2 months of afatinib treatment. $^{13}$ However, whether E709K will impact the sensitivity of EGFR $\mathrm{L} 833 \mathrm{~V} / \mathrm{H} 835 \mathrm{~L}$ to first-generation EGFR-TKIs was unclear. It has been reported that the efficacy of afatinib is superior to first-generation EGFRTKIs in NSCLC harboring uncommon EGFR mutations, such as G719X and L861Q. ${ }^{14,15}$ Recently, some clinical data showed that afatinib may be effective for other rare mutations, such as exon 18 delE709_T710insD and L858M/L861Q compound mutation. ${ }^{16,17}$ In this study, we found that afatinib could be a first-line treatment option in NSCLC patients with L833V/H835L mutations.

It is worth mentioning that the application of NGS provided an opportunity of targeted therapy for the present patient because the traditional ARMS-PCR would not normally detect the $\mathrm{L} 833 \mathrm{~V} / \mathrm{H} 835 \mathrm{~L}$ or other rare mutations. In addition, the $\mathrm{L} 833 \mathrm{~V} / \mathrm{H} 835 \mathrm{~L}$ mutation reported in previous studies was mostly detected in tumor tissue. ${ }^{10,13,18}$ In this study, the mutation was successfully detected using the circulating ctDNA extracted from pleural effusion and plasma, indicating that liquid biopsy could be an alternative mediator for mutation analysis in patients who are difficult to get tumor specimens. It has been reported that circulating cell free DNA (ccfDNA) is a prognostic marker in advanced NSCLC. ${ }^{19}$ Under treatment, ctDNA clearance at first evaluation is associated with objective response and longer survival. ${ }^{20}$ In this case, the ctDNA was undetectable after 1 month and 7 months of afatinib treatment accompanied with the imaging changes, suggesting a good efficacy of afatinib.

In conclusion, this case report suggests that EGFR $\mathrm{L} 833 \mathrm{~V} / \mathrm{H} 835 \mathrm{~L}$ are rare but potentially afatinib responsive mutations in NSCLC. Although it is not realistic to design a clinical trial due to the small number of patients, our case provides more evidence for the clinical application of afatinib in this mutation. Inevitably, a few limitations are associated with this study. How the afatinib take effect on this mutation is not known. A well designed in vitro and in silico studies would provide better understanding of its concrete mechanisms.

\section{Ethical Approval}

Institutional approval was not required to publish the case details.

\section{Patient Informed Consent}

Written informed consent was obtained from the patient for the publication of his case details and images.

\section{Acknowledgment}

The authors thank the patient and her family. We also thank the investigators, study coordinators, operation staff and the whole project team who worked on this case. 


\section{Disclosure of Interest}

Tian Qin is an employee of Burning Rock Biotech. The authors declare no other potential conflicts of interest in this work.

\section{References}

1. Graham RP, Treece AL, Lindeman NI, et al. Worldwide frequency of commonly detected EGFR mutations. Arch Pathol Lab Med. 2018;142(2):163-167. doi:10.5858/arpa.2016-0579-CP

2. Dai L, Su X, Lu L, Lv D. Nonsmall cell lung cancer with rare exon 7 p.A289V mutation in the EGFR gene responds to Icotinib treatment: a case report. Medicine. 2018;97(51):e13809. doi:10.1097/ MD.0000000000013809

3. Yang $\mathrm{M}$, Tong $\mathrm{X}, \mathrm{Xu} \mathrm{X}$, et al. Case Report: osimertinib achieved remarkable and sustained disease control in an advanced non-smallcell lung cancer harboring EGFR H773L/V774M mutation complex. Lung Cancer. 2018;121:1-4. doi:10.1016/j.lungcan.2018.04.006

4. An N, Wang H, Zhu H, et al. Great efficacy of afatinib on a patient with lung adenocarcinoma harboring uncommon EGFR delE709_T710insD mutations: a case report. Onco Targets Ther. 2019;12:7399-7404. doi:10.2147/OTT.S221638

5. Yu X, Zhang X, Zhang Z, et al. First-generation EGFR tyrosine kinase inhibitor therapy in 106 patients with compound EGFR-mutated lung cancer: a single institution's clinical practice experience. Cancer Commun. 2018;38(1):51. doi:10.1186/s40880-018-0321-0

6. Kobayashi S, Canepa HM, Bailey AS, et al. Compound EGFR mutations and response to EGFR tyrosine kinase inhibitors. $J$ Thorac Oncol. 2013;8(1):45-51. doi:10.1097/JTO.0b013e3182781e35

7. Tu HY, Ke EE, Yang JJ, et al. A comprehensive review of uncommon EGFR mutations in patients with non-small cell lung cancer. Lung Cancer. 2017;114:96-102. doi:10.1016/j.lungcan.2017.11.005

8. Koopman B, van der Wekken AJ, Ter Elst A, et al. Relevance and effectiveness of molecular tumor board recommendations for patients with non-small-cell lung cancer with rare or complex mutational profiles. JCO Precis Oncol. 2020;4:393-410. doi:10.1200/PO.20.00008

9. Yi S, Zhuang Y, Zhou J, et al. A comparison of epidermal growth factor receptor mutation testing methods in different tissue types in non-small cell lung cancer. Int J Mol Med. 2014;34(2):464-474. doi:10.3892/ijmm.2014.1789

10. Yang TY, Tsai CR, Chen KC, Hsu KH, Lee HM, Chang GC. Good response to gefitinib in a lung adenocarcinoma harboring a heterozygous complex mutation of L833V and H835L in epidermal growth factor receptor gene. J Clin Oncol. 2011;29(16):e468-469. doi: $10.1200 / J C O .2010 .33 .5802$
11. Li M, Zhou CZ, Yang JJ, et al. The in cis compound EGFR mutations in Chinese advanced non-small cell lung cancer patients. Cancer Biol Ther. 2019;20(8):1097-1104.

12. Qin BD, Jiao XD, Yuan LY, et al. The effectiveness of afatinib and osimertinib in a Chinese patient with advanced lung adenocarcinoma harboring a rare triple EGFR mutation (R670W/H835L/L833V): a case report and literature review. Onco Targets Ther. 2018;11:4739-4745. doi:10.2147/OTT.S167346

13. Frega S, Conte P, Fassan M, Polo V, Pasello G. A triple rare E709K and $\mathrm{L} 833 \mathrm{~V} / \mathrm{H} 835 \mathrm{~L}$ EGFR mutation responsive to an irreversible pan-HER inhibitor: a case report of lung adenocarcinoma treated with afatinib. $J$ Thorac Oncol. 2016;11(5):e63-e64. doi:10.1016/j. jtho.2016.01.023

14. Yang JC, Sequist LV, Geater SL, et al. Clinical activity of afatinib in patients with advanced non-small-cell lung cancer harbouring uncommon EGFR mutations: a combined post-hoc analysis of LUX-Lung 2, LUX-Lung 3, and LUX-Lung 6. Lancet Oncol. 2015;16(7):830-838. doi:10.1016/S1470-2045(15)00026-1

15. Tanaka I, Morise M, Kodama Y, et al. Potential for afatinib as an optimal treatment for advanced non-small cell lung carcinoma in patients with uncommon EGFR mutations. Lung Cancer. 2019;127:169-171. doi:10.1016/j.lungcan.2018.11.018

16. Iwamoto $\mathrm{Y}$, Ichihara $\mathrm{E}$, Hara N, et al. Efficacy of afatinib treatment for lung adenocarcinoma harboring exon 18 delE709_T710insD mutation. Jpn J Clin Oncol. 2019;49(8):786-788. doi:10.1093/jjco/ hyz086

17. Saxon JA, Sholl LM, Janne PA. EGFR L858M/L861Q cis mutations confer selective sensitivity to afatinib. J Thorac Oncol. 2017;12 (5):884-889. doi:10.1016/j.jtho.2017.01.006

18. Zhuang Y, Xu J, Ma H, et al. A sequential method of epidermal growth factor receptor mutation detection reduces false negatives: a new case with doublet mutations of L833V and H835L in China. Clin Lung Cancer. 2013;14(3):295-300. doi:10.1016/j. cllc.2012.11.003

19. Song Y, Hu C, Xie Z, et al. Circulating tumor DNA clearance predicts prognosis across treatment regimen in a large real-world longitudinally monitored advanced non-small cell lung cancer cohort. Transl Lung Cancer Res. 2020;9(2):269-279. doi:10.21037/ tlcr.2020.03.17

20. Pecuchet N, Zonta E, Didelot A, et al. Base-position error rate analysis of next-generation sequencing applied to circulating tumor DNA in non-small cell lung cancer: a prospective study. PLoS Med. 2016;13(12):e1002199. doi:10.1371/journal.pmed.1002199
OncoTargets and Therapy

\section{Publish your work in this journal}

OncoTargets and Therapy is an international, peer-reviewed, open access journal focusing on the pathological basis of all cancers, potential targets for therapy and treatment protocols employed to improve the management of cancer patients. The journal also focuses on the impact of management programs and new therapeutic agents and protocols on patient perspectives such as quality of life, adherence and satisfaction. The manuscript management system is completely online and includes a very quick and fair peer-review system, which is all easy to use. Visit http://www.dovepress.com/ testimonials.php to read real quotes from published authors. 